\title{
Time-Resolved Förster Resonance Energy Transfer Analysis of Single-Nucleotide Polymorphisms: Towards Molecular Typing of Genes on Non-Purified and Non-PCR-Amplified DNA
}

\author{
Luca Nardo ${ }^{1}$, Nicola Camera ${ }^{1}$, Edoardo Totè ${ }^{1}$, Maria Bondani ${ }^{2}$, Roberto S. Accolla ${ }^{3} \&$ Giovanna Tosi $^{3}$ \\ ${ }^{1}$ Department of Science and High Technology, University of Insubria, Italy \\ ${ }^{2}$ Institute for Photonics and Nanotechnology, National Research Council, Italy \\ ${ }^{3}$ Department of Surgical and Morphologic Sciences, University of Insubria, Italy \\ Correspondence: Luca Nardo, Department of Science and High Technology, University of Insubria, Via \\ Valleggio, Como 11-22100, Italy. Tel: 39-238-6272. E-mail: luca.nardo@unimib.it
}

Received: November 30, 2012 Accepted: February 28, 2013 Online Published: April 9, 2013

doi:10.5539/jmbr.v3n1p15 URL: http://dx.doi.org/10.5539/jmbr.v3n1p15

\begin{abstract}
Quantitative assessment of the fluorescence resonance energy transfer (FRET) efficiency between chromophores labeling the opposite ends of gene-specific oligonucleotide probes is a powerful tool to detect DNA polymorphisms with single-nucleotide resolution. The FRET efficiency can be most conveniently quantified by applying a time-resolved fluorescence analysis methodology, time-correlated single-photon counting. Recently, we probed by such technique the highly polymorphic DQB1 human gene. Namely, by using a single oligonucleotide probe and acting on non-amplified DNA samples contained in untreated cell extracts, we demonstrated the ability of pursuing unambiguous recognition of subjects bearing the homozygous DQB1-0201 genotype by exploiting the subtle, yet statistically significant, structural differences between the duplex formed by the probe with DQB1-0201 on the one end and duplexes formed with any of the other alleles, on the other end. The relevance of homozygous DQB1-0201 genotype recognition reseeds in the fact that the latter is overexpressed in subjects affected by insulin-dependent diabetes mellitus in north-eastern Italy.

In this article we review our preceding achievements and report on additional in-vitro experiments aimed at characterizing the duplexes obtained by annealing of the DQB1 allelic variants with a second oligonucleotide probe, with the final scope to achieve full genotyping of DQB1 on raw DNA samples by means of cross-combination of the FRET responses of both probes.
\end{abstract}

Keywords: polymorphism, molecular typing, oligonucleotide probe, time-correlated single-photon counting, time-resolved fluorescence resonance energy transfer

\section{Introduction}

Genetic diseases are, strictly speaking, pathologies resulting from site-specific mutations in the genome. However, in other cases the proneness of an individual to manifest non-genetic diseases can still be correlated to the presence of certain allelic variants of one or more genes. A particularly relevant instance is constituted by auto-immune diseases, which are notably correlated to the polymorphisms of the Human Leucocyte Antigen system (HLA). More specifically, insurgence of several auto-immune diseases is correlated with overexpression of certain genotypic variants of the highly polymorphic DQB1 human gene. For instance, in Caucasian population of north-eastern Italy the homozygous DQB1-0201 genotype is over-represented in subjects suffering from insulin-dependent diabetes mellitus (Tosi, 1993). The risk factor of a given subject with respect to contraction of multiple autoimmune diseases might thus be evaluated, and both preventive health care and early diagnosis be subsequently implemented, by means of wide-screen HLA genotyping.

Nowadays, molecular typing of polymorphic genes may be performed with single nucleotide sensitivity by applying several different techniques (Kim, 2007). However, as a rather common feature, virtually all these techniques require the Polymerase Chain Reaction (PCR) as initial step. Although it is out of doubt that PCR has revolutionized biology, it is not the ideal technique to be applied to the screening of vast populations, due to lack of both readiness and cost-effectiveness. During the last decade, molecular typing techniques based on 
exploitation of the differential fluorescence emission of fluorophore-labeled allele-specific oligonucleotide probes upon recognition of their genomic target have been given increasing interest because of their particularly neat response (i.e. high signal-to-noise ratio and low probability of false-positives detection), conferring to fluorescence-based assays notable readiness and ease of interpretation (Tyagi, 1998; Gaylord, 2005; Al Attar, 2008; Andreoni, 2009a, 2009b; Nardo, 2012). The common rationale of the majority of such techniques is that light emission by the fluorophore functionalizing the oligonucleotide probe (hereafter called the fluorescence donor, D) can be quenched by suitable dyes (hereafter referred to as fluorescence acceptors, A) in a strongly donor-acceptor-distance dependent way by virtue of a non-radiative decay mechanism of the $\mathrm{D}$ from the fluorescent excited state, called fluorescence resonance energy transfer (FRET). The latter occurs whenever the absorption spectrum of the $\mathrm{A}$ is significantly superimposed to the fluorescence emission spectrum of the $\mathrm{D}$.

Molecular typing methods have been devised both making use of D-A dual-labeled probes (Tyagi, 1998; Andreoni, 2009a, 2009b; Nardo, 2012), which are virtually non-emitting while not annealed to their genomic target, and exploiting A selectively binding to the genomic DNA to be typed and probes single-labeled with a D whose fluorescence is "switched off" upon target recognition (Gaylord, 2005; Al Attar, 2008). In many instances (Tyagi, 1998), the response of a typing experiment is barely constituted by the presence/absence of a detectable fluorescence signal. Hence, this class of tests is frequently referred to as ON/OFF methods. However, further options are being explored in the last years, based on quantitative analysis of FRET-tuned fluorescence signals (Gaylord, 2005; Al Attar, 2008; Andreoni, 2009a, 2009b; Nardo, 2012). The basic advantage in doing so reseeds in the fact that quantitative evaluation allows at least partial release of the strict boundaries on signal-to-noise ratio required by ON/OFF strategies, thus making the implementation of assays on less pure DNA samples and/or on reduced DNA template concentrations conceivable, and ultimately opening the way to molecular typing of polymorphic genes directly on the unpurified and non-PCR-amplified DNA contained in crude cell lysates. The ability of determining even slight differences in the quantitative fluorescence response should also allow to perform typing with a single probe capable of hybridizing to any of the possible target sequences (i.e. to each possible polymorphic variant of the gene of interest) with sufficient selectivity with respect to other genomic regions. Allele-specific annealing would in the latter case occur through formation of duplexes whose structural details depend on the probe-target degree of complementarity, thus, in the end, from the target sequence. At least, a reduced number of probes with respect to ON/OFF experiments, in which a number of probes equal to the number of allelic variants displayed by the gene to be typed are intrinsically needed, are most likely necessary. This feature promises to substantially reduce the recourse to multiplexed analysis, thus speeding up and making easier the experimental and analytical procedures, as well as allowing working on much reduced template quantities. A first method to quantitatively evaluate FRET efficiencies is based on relative intensity measurements (Gaylord, 2005; Al Attar, 2008). Progresses in this direction have been made by Al Attar et al. (2008) who have provided as early as in 2008 a prove of principle of the feasibility of detecting the polymorphisms of the ABL portion of the BCR-ABL oncogene (Al Attar, 2008). However, this approach is affected by two major limitations: (i) quantitative assessments of the fluorescence intensity require detectors endowed with broad dynamic ranges, whose low quantum efficiency makes PCR amplification hardly avoidable; (ii) application to non-purified genomic material is prevented by the presence of endogenous fluorophores and, even more unavoidably, by the inherent difficulty in determining probe, target, and fluorescent impurities concentrations. Both the above-mentioned drawbacks can be overcome by quantitating the variations in FRET efficiency through measurements of the fluorescence decay time $\tau_{D}$ of D. Indeed, $\tau_{D}$ can be determined by applying a technique named time-correlated single-photon counting (TCSPC). TCSPC works by detecting and timing one photon per excitation pulse at most. The subsequent reconstruction of the statistical distribution of the detection times relative to the excitation pulses yields the fluorescence decay pattern, whose fitting provides the value of $\tau_{D}$. For this reason, TCSPC is intrinsically independent from the D concentration and takes advantage of the usage of Geiger-mode light detectors, such as single-photon avalanche diodes, which are sensitive to even extremely deem fluorescence pulses, virtually reduced to few/single photons.

In 2009 we applied time-resolved FRET analysis to an in-vitro model system consisting in 25-bases oligonucleotides differing by a single $\mathrm{A} \rightarrow \mathrm{T}$ point mutation from each other (Andreoni, 2009a). The latter were made to anneal to a single oligonucleotide probe, dual-labeled at its 5 ' end with a $\mathrm{D}$, namely 5-Dimethoxytrityloxy-5- [N-((tetramethylrhodaminyl)aminohexyl)-3-acrylimido]- 2'-deoxyUridine- 3'[(2cyanoethyl)- (N,N-diisopropyl)] -phosphoramidite (TAMRA), and at its 3' end with a non-emitting A, 4'(4-Nitrophenyldiazo) -2'- methoxy -5'- methoxy - azobenzene -4''-(N-ethyl)-N-ethyl-2-cyanoethyl(N,N-diisopropyl) phosphoramidite (BHQ2). The choice of TAMRA as the fluorescent label was dictated by two considerations. Firstly, it is optimally excited with green-yellow light, which in turn is only slightly absorbed by endogenous fluorophores. Secondly, in aqueous environment its fluorescence decay is single-exponential, which 
considerably simplifies TCSPC data analysis. The probe was perfectly complementary to one of the oligonucleotides, and bore single-nucleotide mismatches with respect to the others. D fluorescence decay distributions were reconstructed for the different duplexes by means of a TCSPC system endowed with $30 \mathrm{ps}$ resolution. The obtained $\tau_{D}$ values differed by at least 3 standard deviations from an oligonucleotide to the other. As sketched in Figure 1, the effect of single nucleotide mismatches at a duplex end (see $S_{1}$ and $S_{3}$ in the figure) was a systematic reduction of the D-A distance with respect to the perfectly matching duplex $\left(\mathrm{S}_{0}\right)$, which we interpreted as unzipping of the lateral bases. Conversely, single nucleotide mismatches at the center of a duplex $\left(\mathrm{S}_{2}\right)$ brought about an increase in the D-A distance, which we interpreted as due to a local unwinding of the double helical structure similar to that induced by intercalation (Nardo, 2008). In summary, time-resolved FRET proved to be sensitive to single-nucleotide mismatches and the response of the $\mathrm{D}$ fluorescence decay seemed to be related to the mismatch position.

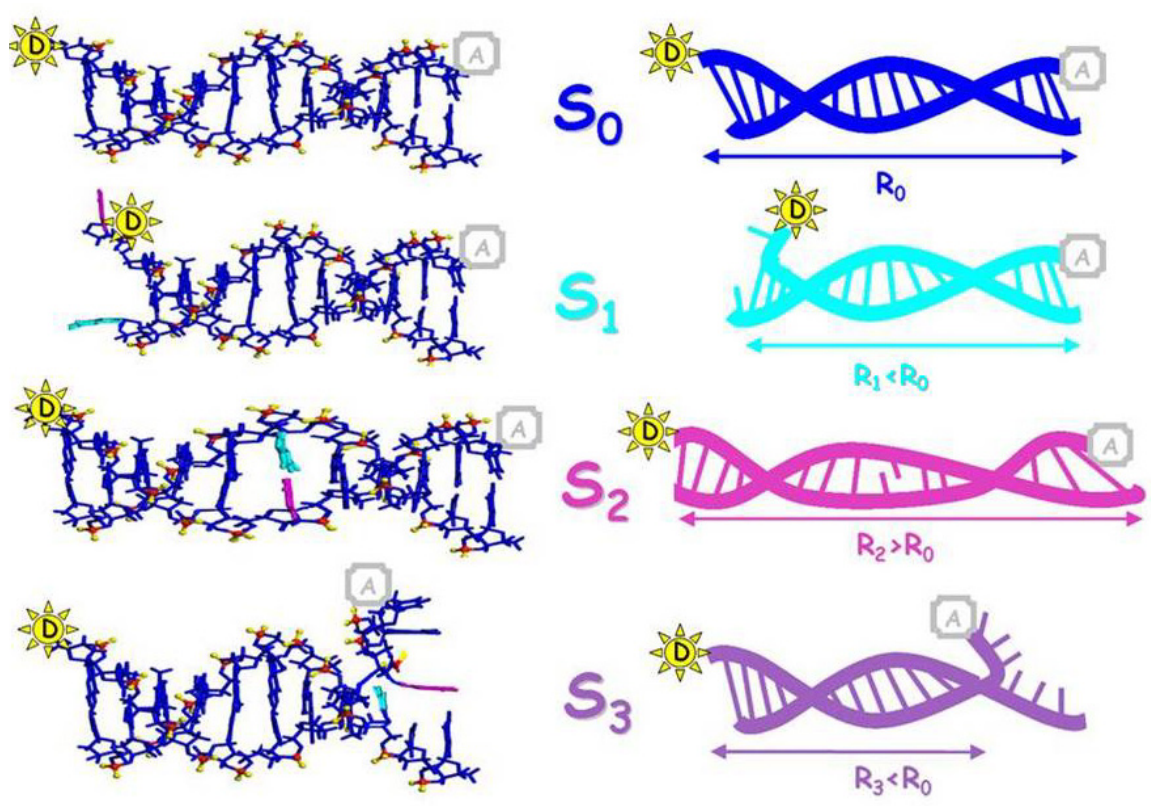

Figure 1. Effects of single-nucleotide mismatches in different positions of a double-stranded oligonucleotide: mismatches at a duplex end (see $\mathrm{S}_{1}$ and $\mathrm{S}_{3}$ ) induce a reduction of the D-A distance with respect to the perfectly matching duplex $\left(\mathrm{S}_{0}\right)$, while single nucleotide mismatches at the center of a duplex $\left(\mathrm{S}_{2}\right)$ increase the D-A distance

The subsequent step towards implementation of a DQB1-typing protocol based on time-resolved FRET was achieved shortly later (Andreoni, 2009b). Our aim was to demonstrate the feasibility of unambiguous recognition of the DQB1-0201 allele, whose presence in homozygous conditions confers, as discussed above, susceptibility to insulin-dependent diabetes mellitus in the population of north-eastern Italy (Tosi, 1993). The sequence of DQB1-0201 is different from that of any other allele in the highly polymorphic trait of the gene encompassing codons 52 to 57. Thus, recognition of that sequence implies recognition of the allele as a whole. For this reason, we decided to elect that region as our genomic target, and to pursue its specific recognition by an oligonucleotide probe with sequence corresponding to that of DQB1-0201 (P0), dual labeled with TAMRA and BHQ2. We used as templates 18-base oligonucleotides mimicking the eight distinct sequences exhibited in the above-mentioned trait by the DQB1 allelic variants. TCSPC measurements were performed for P0 hybridized to each target oligonucleotide. Significantly different $\tau_{D}$ values were determined for the eight duplexes. This result demonstrates that the subtle structural differences among duplexes formed by P0 with each allele may be sensed by applying time-resolved FRET analysis, and that the structures are sufficiently heterogeneous to allow full molecular typing of the target region with this unique probe. However, the selectivity of P0 for recognition of its target trait within the whole genome has proven to be not sufficient to permit application of the protocol on non-amplified genomic templates.

Nonetheless, we recently managed to pursue selective recognition of an extended trait of DQB1, also encompassing codon 51 and the first base of codon 58, by a 22-bases oligonucleotide probe (P1) in 
non-amplified and non-purified DNA samples obtained by bare cell lysis (Nardo, 2012). The results showed the possibility to reveal the presence of the DQB1-0201 allelic variant both in homozygous and heterozygous conditions, and to discriminate within the two cases. However, extension of the probe of a four-base long non-polymorphic trait reduced both the sensitivity of the FRET efficiency to equal D-A distance variations and the heterogeneity of the duplex structures. In conclusion, we failed to obtain a full discrimination of alleles. Namely, even in preliminary calibration measurements undergone on 22-bases oligonucleotide targets, P1 yielded hardly distinguishable $\tau_{D}$ values when annealed to DQB1-05031, DQB1-0602, and DQB1-0402-like sequences.

In this work we report $\tau_{D}$ data obtained by making to hybridize the same oligonucleotides used in (Nardo, 2012) with a different 22-base-pairs oligonucleotide probe (P2). The latter is dual-labeled with the same chromophores used in P0 and P1. However, to the aim of overcoming the ambiguities inherent to the P1 FRET response, the sequence of $\mathrm{P} 2$ is complementary to the DQB1-05031 sequence, while it obviously contains mismatches with respect to both the DQB1-0402-allele and the DQB1-0602-allele mimicking oligonucleotides. Significantly different $\tau_{D}$ values are measured with $\mathrm{P} 2$ in the three instances.

\section{Method}

\subsection{Oligonucleotides and Sample Preparation}

In Figure 2 the sequences of the eight target oligonucleotides reproducing the possible sequences manifested by the 22-bases polymorphic trait encompassing codons 51 to 57 and the first base of codon 58 are depicted. In the same figure, the TAMRA-BHQ2 dual-labelled probes P1 and P2, with sequences complementary to the DQB1-0201 and DQB1-05031 allele mimicking oligos, respectively, are also shown. Mismatch-sites of the target oligonucleotides with respect to P1 are black-shaded, while those with respect to $\mathrm{P} 2$ are light-gray shaded. Mismatching sites common to both probes are indicated by dark-grey shading. The unlabelled oligonucleotides were purchased by TIB Molbiol (Genova, Italy) and were desalted and further purified by double RP procedure.

\begin{tabular}{|c|c|c|c|c|c|c|c|c|c|c|c|c|c|c|c|c|c|c|c|c|c|c|c|c|}
\hline 0201 & 5'- & $\mathrm{T}$ & G & C & G & A & C & G & A & $\mathrm{C}$ & C & C & C & G & A & C & $\mathrm{G}$ & $\mathrm{G}$ & A & C & G & G & C & -3 \\
\hline 0501 & 5'- & $\mathrm{T}$ & G & C & G & $\mathbf{G}$ & $\mathrm{C}$ & G & $\mathrm{T}$ & C & C & C & C & G & C & C & G & G & A & C & $\mathbf{A}$ & $\mathbf{A}$ & C & -3 \\
\hline 0502 & 5'- & $\mathrm{T}$ & $\mathrm{G}$ & $\mathrm{C}$ & G & $\mathbf{G}$ & $\mathrm{C}$ & G & $\mathrm{T}$ & $\mathrm{C}$ & C & C & C & G & C & C & $\mathrm{G}$ & $\mathrm{G}$ & A & $\mathrm{T}$ & C & G & C & -3 \\
\hline 05031 & $5 '-$ & $\mathrm{T}$ & G & C & G & $\mathbf{G}$ & $\mathrm{C}$ & G & $\mathrm{T}$ & $\mathrm{C}$ & C & C & $\mathrm{C}$ & G & C & C & $\mathrm{G}$ & G & $\mathrm{A}$ & C & $\mathrm{T}$ & G & C & $-3^{\prime}$ \\
\hline 0602 & 5'- & $\mathrm{T}$ & G & C & $\mathrm{G}$ & G & C & G & $T$ & $\mathrm{C}$ & C & C & C & G & C & C & $\mathrm{G}$ & $\mathrm{G}$ & A & $\mathrm{C}$ & $\mathrm{T}$ & $\mathbf{A}$ & C & -3 \\
\hline 0301 & 5'- & $\mathrm{T}$ & $\mathrm{G}$ & C & G & $\mathbf{G}$ & C & $\mathrm{G}$ & A & C & C & C & C & G & G & C & $G$ & $\mathrm{G}$ & $\mathrm{A}$ & C & $\mathbf{T}$ & $\mathrm{G}$ & C & -3 \\
\hline 0302 & 5'- & $\mathrm{T}$ & G & C & G & $\mathbf{G}$ & $\mathrm{C}$ & G & A & $\mathrm{C}$ & C & C & $\mathrm{C}$ & G & G & C & $\mathrm{G}$ & $\mathrm{G}$ & A & C & G & G & C & -3 \\
\hline 0402 & $5 '-$ & $\mathrm{T}$ & G & C & G & $\mathbf{G}$ & $\mathrm{C}$ & $\mathrm{G}$ & A & $\mathrm{C}$ & C & C & C & G & C & C & $\mathrm{G}$ & A & A & $\mathrm{C}$ & $T$ & $\mathrm{G}$ & $\mathrm{C}$ & -3 \\
\hline Probe 1 & $5 '-$ & A & C & $\mathrm{G}$ & C & $\mathrm{T}$ & $\mathrm{G}$ & C & $\mathrm{T}$ & $\mathrm{G}$ & $\mathrm{G}$ & G & G & C & $\mathrm{T}$ & $\mathrm{G}$ & C & $\mathrm{C}$ & $\mathrm{T}$ & G & $\mathrm{C}$ & C & G & $-3^{\prime}$ \\
\hline Probe 2 & 5'- & A & C & G & C & C & G & C & A & G & G & G & G & C & G & G & C & C & $\mathrm{T}$ & G & A & C & G & -3 \\
\hline Codons & $5 \%$ & & 51 & & & 52 & & & 53 & & & 54 & & & 55 & & & 56 & & & 57 & & & $-3^{\prime}$ \\
\hline
\end{tabular}

Figure 2. Sequences of the eight target oligonucleotides and of the TAMRA-BHQ2 dual-labelled probes P1 and P2. Mismatch-sites of the target oligonucleotides with respect to P1 are black-shaded, those with respect to P2 are light-gray shaded. Mismatching sites common to both probes are indicated by dark-grey shading

The dual-labelled probes were purchased by Eurofins MWG Operon (Ebersberg, Germany) and purified by high-performance liquid chromatography. A nominal amount of twenty nanomoles of the lyophilized oligonucleotides was resuspended in appropriate volumes (indicated by the suppliers) of Tris-HCl EDTA buffer at $\mathrm{pH} 7.6$ and $10 \mathrm{mM}$ ionic strength, to obtain $5 \mu \mathrm{M}$ concentrated solutions. Equal amounts $(100 \mu \mathrm{L})$ of probe and target stock solutions were mixed to obtain the solutions submitted to the annealing protocol. The latter, which is fully described elsewhere, consisted in heating the samples to $98^{\circ} \mathrm{C}$ in order to denaturate any residual secondary structures of the single-stranded oligonucleotides, cooling at the rate of $1{ }^{\circ} \mathrm{C} / \mathrm{min}$ to the optimal annealing temperature, derived from the melting temperature of the perfectly matching duplex as described in (Nardo, 2012), and letting the probe react with the (more or less) complementary oligonucleotide for 20 minutes. Finally, the solutions were cooled to $25^{\circ} \mathrm{C}$ at $3^{\circ} \mathrm{C} / \mathrm{min}$ rate. The thermostat of a ThermoQuest-Finnigan gas chromatograph (San Jose, CA, USA) was used as the temperature controller. 
The obtained solutions were diluted to a final probe concentration of $250 \mathrm{nM}$, to produce the sample for TCSPC analysis.

\subsection{Time-Correlated Single-Photon Counting Apparatus}

The fluorescence excitation/detection apparatus is extensively described elsewhere (Andreoni, 2009b). Briefly, a $113 \mathrm{MHz}$ repetition rated train of excitation pulses (pulse duration $6.4 \mathrm{ps}$ ) at $532 \mathrm{~nm}$ is provided by a Nd: VAN cw-mode-locked laser (GE-100, Time Bandwidth Products, Zurich, CH) and suitably attenuated (typically by a factor of $>10^{3}$ ) by means of neutral density filters, in order to achieve single-photon regime. Fluorescence is collected at $90^{\circ}$ to the excitation beam through a long-wavelength-pass filter with cut at $550 \mathrm{~nm}$, and focused on the sensitive area of a single-photon avalanche diode (PDM50, Micro-photon-devices, Bolzano, IT) by a 20X microscope objective. The avalanche pulses give the START signal to an integrated TCSPC board (which is a single card of a SPC 152 module, Becker \& Hickl, Berlin, DE). The STOP signal is provided by the subsequent laser pulse, as detected by a fast photodiode internal to the laser. The START-STOP lag times occurring in a time window of $9 \mathrm{~ns}$ are digitized with $2.44 \mathrm{ps} / \mathrm{channel}$ resolution. The full-width at half-maximum duration of the detected excitation pulse is $<30 \mathrm{ps}$.

\subsection{Data Analysis}

Due to satisfactory yield of the annealing protocol, the data were treated as those obtained on the oligonucleotide samples of (Nardo, 2012). The data analysis procedure is explained in details in the quoted reference. Briefly, the absorbance of each sample at the TAMRA absorption peak $(556 \mathrm{~nm})$ was preliminarily measured. Each fluorescence decay histogram was acquired up to the fixed value of 65535 counts at the peak and the acquisition time measured. The absorption spectrum and decay distribution of a solution containing only the probe, at 250 $\mathrm{nM}$ nominal concentration, were also acquired. Each experimental decay pattern was normalized with respect to absorbance and acquisition time. The normalized decay obtained for the pure-probe sample was fitted to a three-exponential decay model, see Equation (1):

$$
F(t)=y_{0}+A_{1} \exp \left[\left(t_{0}-t\right) / \tau_{1}\right]+A_{2} \exp \left[\left(t_{0}-t\right) / \tau_{2}\right]+A_{3} \exp \left[\left(t_{0}-t\right) / \tau_{3}\right]
$$

In the above equation, besides the decay times $\tau_{i}$ and pre-exponential factors $A_{\mathrm{i}}$, two other parameters appear, namely $y_{0}$ and $t_{0}$. The former prepresents a constant offset added to the decay function, accounts for the both the dark-counts of the detectors (START signals provided by thermally induced avalances) and the detection of environmental non-time-correlated light, and was let free to vary during the fit. The latter represents the instant in which the excitation pulse impinges on the sample, and was fixed to the time-lag corresponding to the peak channel. The longest-lived component in the pure-probe decay is attributed to the emission of unquenched probe molecules and/or residual free TAMRA, whose fraction relative to the total TAMRA concentration is constant in all the samples. Thus, after synchronization of the histograms to the corresponding peak channels, we got rid of the unquenched TAMRA contribution to the decay of duplex samples by simply subtracting from each duplex histogram an exponential component with the same lifetime and pre-exponential factor as those derived for the longest-lived transient resolved in the pure-probe sample decay pattern. The background value $y_{0}$ was also subtracted to all the decay distributions. The $\tau_{D}$ values tagging the conformations of the different duplexes were derived by performing single-exponential fits of the so-obtained decay patterns according to Equation (2):

$$
F(t)=A \exp \left[(t) / \tau_{D}\right]
$$

\section{Results and Discussion}

In a previous article (Nardo, 2012) the time-resolved FRET methodology was presented as a valid technique to recognize allelic-variants of a human gene (namely DQB1) by means of a D-A dual-labeled oligonucleotide probe (namely P1) capable of selectively hybridize to a 22-bases trait of DQB1 in unpurified and non-PCR amplified genomic DNA samples consisting in crude cell extracts. The allelic variants were tagged through determination of the decay time $\tau_{D}$ of the $\mathrm{D}$ fluorophore, which proved to be sensitive to the specificities of the probe-template duplex conformation. The $\tau_{D}$ values derived for P1 are reported in Table 1. As made apparent by straightforward comparison of the $\tau_{D}$ differences between different alleles with the experimental uncertainties in the $\tau_{D}$ values determination, most of the allelic sequences could be safely discriminated by using uniquely P1 as the probe. In particular, we specifically intended to recognize the IDDM-correlated DQB1-0201 allele. Hybridization of P1 to the latter yielded the $\tau_{D}$ value $\tau_{D}=2725 \pm 3 \mathrm{ps}$, while the least differing $\tau_{D}$ value was obtained in the case of the DQB1-0302 allele (which was the least different in sequence with respect to DQB1-0201). In the latter case we measured $\tau_{D}=2599 \pm 3 \mathrm{ps}$. The difference between the two $\tau_{D}$ values was as large as 42 standard deviations, corresponding to negligibly small probability of incurring in "false positive" recognition of DQB1-0201. More in general, the ability of discriminating among the possible homozygous 
genotypes by means of single $\tau_{D}$ measurements upon annealing with $\mathrm{P} 1$ can be evaluated under the very reasonable assumption of stochastic (i.e. Gaussian) distribution of the variable $\tau_{D}$. If the latter assumption holds, the probability of getting an experimental value for $\tau_{D}$ different from the average values reported in Table 1 by more than $3 \sigma$ is $\approx 0.3 \%$. Similarly, the genotype DQB1-0YYY is erroneously attributed to a DQB1-0XXX homozygous DNA template whenever a value of $\tau_{D}$ more similar to $\tau_{D, Y Y Y}$ than to $\tau_{D, X X X}$ is measured. The probability of such an event is determined by the value assumed by the "reliability parameter" $\Delta \tau_{D} / 2 \sigma$. Namely, it is $<0.3 \%$ if $\Delta \tau_{D} / 2 \sigma>3$. Thus, in spite of the tiny $\tau_{D}$ differences between certain allelic variants, reliable typing is assured by the high repeatability of the $\tau_{D}$ measurements. Two exemplary instances of the above statement are offered by alleles DQB1-0301 and DQB1-0402 $\left(\Delta \tau_{D}=19 \mathrm{ps}\right)$ on the one end, and alleles DQB1-0301 and DQB1-0501 $\left(\Delta \tau_{D}=33 \mathrm{ps}\right)$. For the first pair of alleles, $\Delta \tau_{D}$ is comparable with the TCSPC system temporal resolution, whilst for the second one it is even lower. However, $\sigma\left(\tau_{D}\right)=3$ ps for any of the above situations. Consequently, the probability of attributing erroneously the DQB1-0301 sequence to a DQB1-0402 template is $\approx$ $0.3 \%\left(\Delta \tau_{D} / 2 \approx 3 \sigma\right)$, while we get an even lower probability of confusing DQB1-0301 with DQB1-0501 $\left(\Delta \tau_{D} / 2 \approx\right.$ $5.5 \sigma$ ). On the contrary, it was impossible to distinguish between DQB1-05031 and DQB1-0602 decay times, as $\Delta \tau_{D} / 2<\sigma$. Moreover, discrimination between the latter alleles and DQB1-0502 was somewhat tricky, as $\Delta \tau_{D} / 2 \approx$ $\sigma$, meaning that the probability of mis-assignment was $\approx 33 \%$.

Table 1. Values of $\tau_{D}$ determined for duplexes formed by the different DQB1-alleles-mimicking oligonucleotides with P1 (second column) and P2 (third column). The uncertainties are expressed in terms of the standard deviation among three parallels

\begin{tabular}{lll}
\hline DQB1-Allele-mimicking oligo & $\tau_{D}(\mathrm{P} 1) \pm \sigma\left(\tau_{D}\right)[\mathrm{ps}]^{*}$ & $\tau_{D}(\mathrm{P} 2) \pm \sigma\left(\tau_{D}\right)[\mathrm{ps}]$ \\
\hline DQB1-0201/0201 & $2725 \pm 3$ & $2563 \pm 8$ \\
DQB1-0501/0501 & $2514 \pm 3$ & $2201 \pm 13$ \\
DQB1-0502/0502 & $2404 \pm 11$ & $2030 \pm 3$ \\
DQB1-05031/05031 & $2427 \pm 2$ & $2397 \pm 2$ \\
DQB1-0602/0602 & $2430 \pm 9$ & $2194 \pm 5$ \\
DQB1-0301/0301 & $2481 \pm 3$ & $2137 \pm 4$ \\
DQB1-0302/0302 & $2599 \pm 3$ & $2489 \pm 3$ \\
DQB1-0402/0402 & $2462 \pm 3$ & $1969 \pm 1$ \\
\hline
\end{tabular}

*Data reproduced from (Nardo, 2012).

We now examine the results of similar experiments performed by using P2 as the oligonucleotide probe. The $\tau_{D}$ values obtained in the present case are listed in the last column of Table 1. They are obviously different from those obtained with $\mathrm{P} 1$, because of the different mismatches. By means of these values it is impossible to discriminate between allele DQB1-0501 and DQB1-0602. Thus, complete typing of DQB1 is not feasible also by using $\mathrm{P} 2$ as the unique probe. However, a cross-correlated analysis of the results obtained with both probes allows removal of any ambiguity in the attribution of the correct genotype to homozygous DQB1 samples. Namely, as shown in Figure 3a), the D fluorescence decay pattern is notably different for P2 hybridized to either DQB1-05031 (grey dots, the pertaining best single-exponential fitting curve is indicated by the black line) or DQB1-0602 (black circles, the pertaining best single-exponential fitting curve is indicated by the red line). We recall that the latter two alleles were indistinguishable with P1, as shown by the pertaining decay distributions reported in Figure $3 \mathrm{~b}$ ), which, in spite of the zoomed scale, are almost superimposed. By using P2, the reliability parameter calculated in this instance takes the value $\Delta \tau_{D} / 2 \sigma \approx 20$ (by considering the higher standard deviation associated to determination of $\tau_{D, 0602}$ ). Furthermore, the $\tau_{D}$ value obtained for P2 hybridized to DQB1-0502 differs from that measured in the case of DQB1-0602 by 33 standard deviations, and is even more different from that measured for DQB1-05031. 

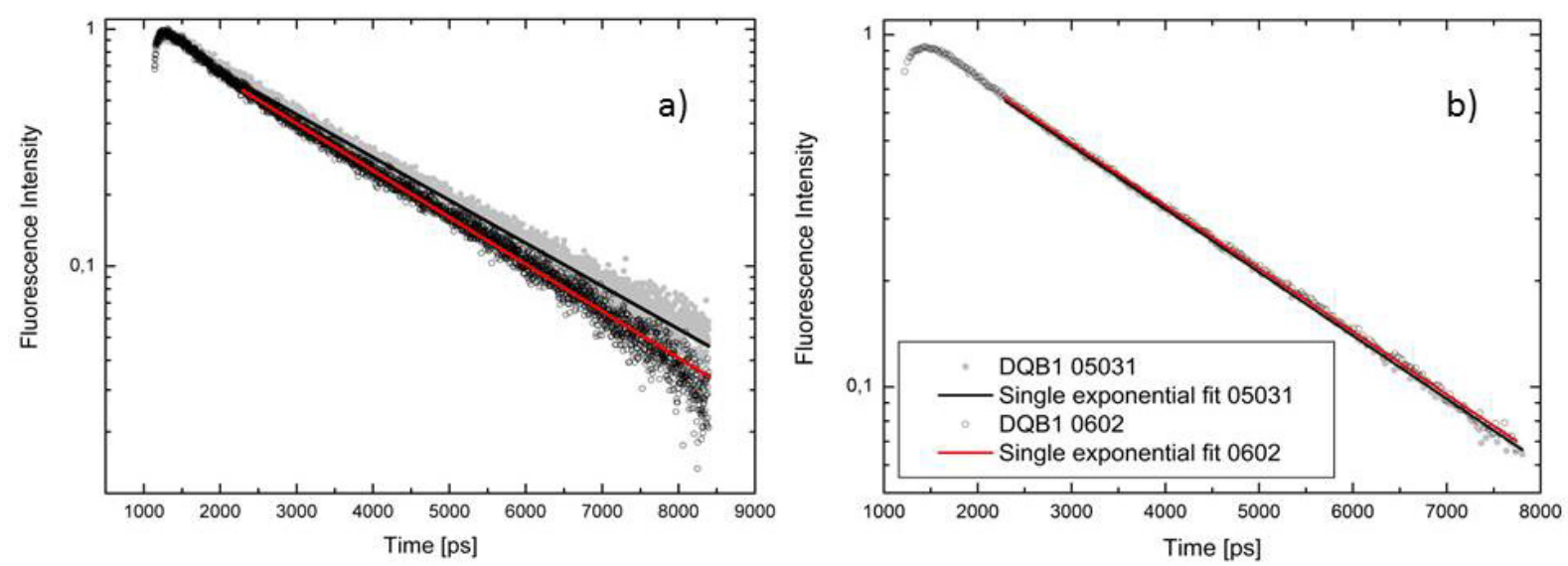

Figure 3. Fluorescence decay patterns of TAMRA labelling a) P2 and b) P1 hybridized to the oligos mimicking the DQB1-05031 (gray dots) and DQB1-0602 (black circles) allelic sequences in the trait encompassing codons 51-57 and the first base of codon 58. Solid lines indicate the corresponding single exponential decay best-fitting curve (see Equation 2 in the text)

In conclusion, we managed to pursue statistically reliable discrimination of each allelic variant of a highly polymorphic trait of the DQB1 human gene by combining the results of time-resolved FRET experiments performed by using two different D-A oligonucleotide probes. The above experiments have been successfully implemented on non-PCR amplified and non-purified DNA templates with one of the probes. The selectivity of the second probe in recognizing its genomic target in similarly raw samples is being evaluated. The performances of the method in typing heterozygous DQB1 genotypes are also currently under investigation.

\section{Acknowledgements}

The authors gratefully acknowledge the contribution of Prof. Alessandra Andreoni to fruitful discussions on the manuscript. We also thank Dr. Angelo Maspero for assistance during the annealing procedures. The postdoctoral fellowship of Dr. Luca Nardo has been funded by the project POR FSE Regione Lombardia, Ob. 2 Asse IV 2007-2013, through an FP7 cooperation programme.

\section{References}

Al Attar, H. A., Norden, J. O’Brien, S., \& Monkman, A. P. (2008). Improved single nucleotide polymorphism detection using conjugated polumer/surfactant system and peptide nucleic acid. Biosens. Bioelectron., 23, 1466-1472. http://dx.doi.org/10.1016/j.bios.2008.01.005

Andreoni, A., Bondani, M., \& Nardo, L. (2009a). Feasibility of single nucleotide polymorphism genotyping with a single probe by time-resolved Forster resonance energy transfer. Mol. Cell. Probes, 23, 119-121. http://dx.doi.org/10.1016/j.mcp.2008.12.008

Andreoni, A., Bondani, M., \& Nardo, L. (2009b). Time-resolved FRET method for typing polymorphic alleles of the human leukocyte antigen system by using a single DNA probe. Photochem. Photobiol. Sci., 8, 1202-1206. http://dx.doi.org/10.1039/b906043j

Gaylord, B. S., Massie, M. R., Feinstein, S. C., \& Bazan, G. C. (2005). SNP detection using peptide nucleic acid probes and conjugated polymers: applications in neurodegenerative disease identification. Proc. Natl. Acad. Sci. USA, 102, 34-39. http://dx.doi.org/10.1073/pnas.0407578101

Kim, S., \& Misra, A. (2007). SNP genotyping: Technologies and biomedical applications. Annu. Rev. Biomed. Eng., 9, 289-320. http://dx.doi.org/10.1146/annurev.bioeng.9.060906.152037

Nardo, L., Bondani, M., \& Andreoni, A. (2008). DNA-ligand binding-mode discrimination by characterizing fluorescence resonant energy transfer trough lifetime measurements with picosecond resolution. Photochem. Photobiol., 84, 101-110. http://dx.doi.org/10.1111/j.1751-1097.2007.00204.x

Nardo, L., Tosi, G., Bondani, M., Accolla, R. S., \& Andreoni, A. (2012). Typing of a polymorphic human gene conferring susceptibility to insulin-dependent diabetes mellitus by picosecond-resolved FRET on non-purified/non-amplified genomic DNA. DNA Res., 19, 347-355. http://dx.doi.org/10.1093/dnares/dss017

Tosi, G., Mantero, G., Magalini, A. R., Primi, D., Soffiati, M., Pinelli, L., ... Accolla, R. S. (1993). HLA-DQB1 
typing of north-east Italian IDDM patients using amplified DNA, oligonucleotide probes, and a rapid DNA-enzyme immunoassay (DEIA). Mol. $\quad$ Immunol., $\quad 30, \quad 69-76$. http://dx.doi.org/10.1016/0161-5890(93)90427-D

Tyagi, S., Bratu, D. P., \& Russel Kramer, F. (1998). Multicolor molecular beacons for allele discrimination. Nat. Biotechnol., 16, 49-53. http://dx.doi.org/10.1038/nbt0198-49 